\title{
Real-World Outcomes of Nivolumab in Patients With Unresectable Hepatocellular Carcinoma in an Endemic Area of Hepatitis B Virus Infection
}

OPEN ACCESS

Edited by:

Ka on Lam,

The University of Hong Kong,

Hong Kong

Reviewed by:

Daniel Lin,

Thomas Jefferson University,

United States

Johan Nicolay Wiig,

Oslo University Hospital, Norway

*Correspondence:

Pil Soo Sung

pssung@catholic.ac.kr

Jeong Won Jang

garden@catholic.ac.kr

Seung Kew Yoon

yoonsk@catholic.ac.kr

Specialty section:

This article was submitted to Gastrointestinal Cancers,

a section of the journal

Frontiers in Oncology

Received: 20 March 2020

Accepted: 26 May 2020

Published: 30 June 2020

Citation:

Sung PS, Jang JW, Lee J, Lee SK, Lee HL, Yang H, Nam HC, Lee SW, Bae SH, Choi JY, Han NI and Yoon SK

(2020) Real-World Outcomes of

Nivolumab in Patients With

Unresectable Hepatocellular

Carcinoma in an Endemic Area of

Hepatitis B Virus Infection.

Front. Oncol. 10:1043.

doi: 10.3389/fonc.2020.01043

\begin{abstract}
Pil Soo Sung ${ }^{1,2 *}$, Jeong Won Jang ${ }^{1,2 *}$, Jaejun Lee ${ }^{1,3}$, Soon Kyu Lee ${ }^{1,2}$, Hae Lim Lee ${ }^{1,4}$, Hyun Yang ${ }^{1,3}$, Hee Chul Nam 1,2, Sung Won Lee ${ }^{1,4}$, Si Hyun Bae ${ }^{1,3}$, Jong Young Choi 1,2, Nam Ik Han ${ }^{1,4}$ and Seung Kew Yoon ${ }^{1,2 *}$

${ }^{1}$ The Catholic University Liver Research Center, College of Medicine, The Catholic University of Korea, Seoul, South Korea, ${ }^{2}$ Division of Gastroenterology and Hepatology, Department of Internal Medicine, College of Medicine, Seoul St. Mary's Hospital, The Catholic University of Korea, Seoul, South Korea, ${ }^{3}$ Division of Gastroenterology and Hepatology, Department of Internal Medicine, College of Medicine, Eunpyeong St. Mary's Hospital, The Catholic University of Korea, Seoul, South Korea, ${ }^{4}$ Division of Gastroenterology and Hepatology, Department of Internal Medicine, College of Medicine, Bucheon St. Mary's Hospital, The Catholic University of Korea, Seoul, South Korea
\end{abstract}

Real-world results of nivolumab monotherapy against HCC are lacking in the hepatitis $\mathrm{B}$ virus (HBV)-endemic, Asia-Pacific regions. Moreover, heterogeneous responses to immune checkpoint inhibitors have rarely been described in advanced HCC. The aim of this study is to evaluate the efficacy and safety of nivolumab monotherapy in a real-world setting in 33 Korean patients with unresectable HCC. In our cohort, twenty-nine patients $(88 \%)$ showed HBsAg positivity. At the time of nivolumab initiation, 4 among 33 patients (12\%) were classified as Barcelona Clinic Liver Cancer (BCLC)-B stage and 29 (88\%) as BCLC-C stage, respectively. Prior sorafenib treatment was given to 31 (94\%) patients, and $13(39 \%)$ received prior regorafenib treatment. For the liver reserve, patients were classified as Child-Pugh class A (79\%) and B (21\%), respectively. Grade 3 toxicities occurred in one patient, who developed pneumonitis after 5 cycles of nivolumab treatment. Best overall responses were complete response in 2 patients out of the 33 enrolled patients (6\%), partial response in 4 patients (12\%) and stable disease in 4 patients (12\%). With 29 patients having images for the response evaluation, the objective response rate was $21.4 \%$. The median overall survival (OS) of the cohort was 26.4 weeks (range 2.3-175.1). Achieving objective responses, pre-treatment small tumors (maximal diameter $<5 \mathrm{~cm}$ ) and favorable liver function as assessed by Albumin-Bilirubin grade were significant factors for the favorable OS. Interestingly, differential responses to nivolumab among multiple tumors in a single patient were noted in 6 patients (18\%). In these patients, small metastatic tumors were regressed, although their larger tumors did not respond to nivolumab monotherapy. In summary, nivolumab treatment seems clinically efficacious in treating unresectable HCC in an endemic area of HBV infection. Further prospective evaluation is required to overcome the heterogeneous efficacy of nivolumab monotherapy according to the baseline tumor burden.

Keywords: hepatocellular carcinoma, nivolumab, objective response, tumor size, tumor heterogeneity 


\section{INTRODUCTION}

Worldwide, hepatocellular carcinoma (HCC) is the fourth most common cause of cancer-related mortality. There are more than 850,000 new cases of liver cancer annually, $90 \%$ of which are HCC (1). Risk factors for HCC include chronic hepatitis B (CHB) and chronic hepatitis $\mathrm{C}(\mathrm{CHC})$, non-alcoholic fatty liver disease (NAFLD) and excessive alcohol ingestion (1). In Asian countries, where hepatitis B virus (HBV) infection is prevalent and accounts for $80 \%$ of victims, a considerable number of patients receive only supportive care or, at best, palliative treatments (2).

Sorafenib, a multi-tyrosine kinase inhibitor, has been the only drug available in the last decade to combat HCC (3). Recently, three tyrosine kinase inhibitors have demonstrated improved outcomes: lenvatinib in the first-line, and regorafenib and cabozantinib after first-line failure (4). Although they showed some promising results in terms of efficacy, their use may be limited due to the adverse effects and the potential decrease in the liver reserve. Immune checkpoint inhibitors are intended to target the programmed cell death protein-1 (PD-1), programmed death-ligand 1 (PD-L1), or cytotoxic T lymphocyte-associated protein-4 (CTLA-4) (5). Clinical trials with nivolumab and pembrolizumab in unresectable HCC, representative anti-PD-1 antibodies, had been anticipated to show prolonged survivals in patients treated with these drugs. However, only 14 to $18 \%$ of patients treated with pembrolizumab or nivolumab monotherapy had objective tumor responses (6-8). More recently, a phase 3 randomized, multi-centre study (CheckMate 459) evaluating nivolumab monotherapy versus sorafenib as a first-line treatment of unresectable HCC, did not achieve its primary endpoint of overall survival (OS) $(9,10)$. Moreover, unlike other solid tumors, there was no marked association identified between the levels of tumor cell PD-L1 expression and responses to nivolumab in HCC, reported by earlier Keynote-224 and CheckMate-040 studies $(5,11,12)$. Currently, there are no validated biomarkers for HCC immunotherapy (13).

Recent sub-analysis of the CheckMate-040 study between intent-to-treat (ITT) overall population and an Asian cohort with prior sorafenib failure showed that treatment responses of Asian patients were similar to those of the overall population (14). This disappointing performance of nivolumab monotherapy may be attributed to the immune heterogeneity of $\operatorname{HCC}(15,16)$. However, there is a lack of real-world clinical data demonstrating the heterogeneous responses to nivolumab. This study aims to evaluate the efficacy and safety of nivolumab monotherapy by performing retrospective analyses of patient data. The data was collected from HCC patients attending three university-affiliated hospitals in Korea where HBV infection is endemic. Specifically, we focused on the responses to nivolumab monotherapy, for every tumor in a single patient, to identify the factors associated with the heterogeneous responses to this treatment.

\section{METHODS}

\section{Study Design and Population}

The Institutional Review Board of The Catholic University of Korea approved this study (Xc20RIDI0015), which was carried out in accordance with the Declaration of Helsinki. Data was collected between October 2016 and November 2019 from 33 consecutive patients treated at three universityaffiliated hospitals in Korea. Among the enrolled patients, 31 patients were enrolled between February 2018 and November 2019. All patients had a verified diagnosis of unresectable HCC by updated international guidelines $(17,18)$ and were treated with nivolumab. Experienced hepatologists reviewed the patients' medical data. The survival data of the patients continued to be followed-up until February 2020. Survival was determined to be from the point of commencing nivolumab treatment until the final follow-up or until the patient died, regardless of the cause. The inclusion criteria were a diagnosis of the inoperable HCC treated with nivolumab. Albumin-Bilirubin (ALBI) grade (19) was calculated to determine the liver reserve of patients treated with nivolumab.

\section{Nivolumab Treatment and Response Evaluation}

Each patient received an intravenously delivered dose of $3 \mathrm{mg} / \mathrm{kg}$ nivolumab (OPDIVO $\AA$, Bristol-Myers Squibb) every two weeks. Every 4 to 8 weeks during treatment, full blood counts were performed, and a number of markers were evaluated including alpha-fetoprotein (AFP), alanine aminotransferase (ALT), bilirubin, and prothrombin time. Nivolumab was administered according to the recommended dose and safety information. Where necessary, doctors would adjust the treatment schedules. Toxicities of nivolumab were diagnosed and managed as previously described $(20,21)$.

Using the modified Response Evaluation Criteria in Solid Tumors (mRECIST) tool, two independent radiologists assessed the response to the treatment, as described elsewhere (3). A maximum of two lesions per organ and five in total were chosen for the evaluation of the treatment responses by mRECIST $(3,22)$. Extrahepatic tumors exhibiting enhanced contrast were considered as target lesions, whereas macroscopic vascular invasions were regarded to be non-target lesions. The mRECIST tool categorizes a complete response (CR) when the intratumoural arterial enhancement disappears from all tumors. A partial response (PR) is defined when the sum of the diameters of enhanced lesions are reduced by no $<30 \%$. However, if the sum of the diameters of enhancing lesions increases by $20 \%$ or more, the disease is categorized as progressive (PD). Disease states not categorized part of $\mathrm{PD}$ and $\mathrm{PR}$, were determined to be stable $(\mathrm{SD})(3,22)$. The sum of $\mathrm{CR}, \mathrm{PR}$ and $\mathrm{SD}$ rates formed the disease control rate (DCR). The response evaluation was conducted regularly, between two and four nivolumabtreatment cycles.

\section{Statistical Analysis}

For the statistical analyses, SPSS version 26 software (IBM Corp., Armonk, NY, USA) was used. A chi-square test was used to analyse the two groups' categorical variables, and an independent $t$-test was conducted to evaluate the continuous variables. Univariate and multivariate analyses were performed 
TABLE 1 | Clinical parameters of study patients.

\begin{tabular}{|c|c|}
\hline Clinical parameters & $n=33$ \\
\hline Median age (range) & $57(37-79)$ \\
\hline Sex (male), $n$ (\%) & $25(75.8)$ \\
\hline HBsAg-positivity, $n$ (\%) & $29(87.9)$ \\
\hline Anti-HCV-positivity, $n$ (\%) & $1(3)$ \\
\hline Median tumor size, $\mathrm{cm}$ & 3.5 \\
\hline$<5 \mathrm{~cm}, n(\%)$ & $21(64)$ \\
\hline$\geq 5 \mathrm{~cm}, n(\%)$ & $12(36)$ \\
\hline Multiple tumors, $n$ (\%) & $33(100)$ \\
\hline Portal vein tumor thrombosis, $n(\%)$ & $10(30)$ \\
\hline Extrahepatic metastasis, $n$ (\%) & $26(79)$ \\
\hline BCLC stage B/C, $n(\%)$ & 4/29 (12/88) \\
\hline Median AFP (range), ng/mL & $665(1.3-160000)$ \\
\hline <1000 ng/mL, n (\%) & $17(52)$ \\
\hline$\geq 1000 \mathrm{ng} / \mathrm{mL}, n(\%)$ & $16(49)$ \\
\hline \multicolumn{2}{|l|}{ Child-Pugh score } \\
\hline $5, n(\%)$ & $20(61)$ \\
\hline $6, n(\%)$ & $6(18)$ \\
\hline $7, n(\%)$ & $7(21)$ \\
\hline ALBI grade $1 / 2 / 3, n(\%)$ & $15 / 18 / 0(45 / 55 / 0)$ \\
\hline \multicolumn{2}{|l|}{ Prior therapy to nivolumab, $n$ (\%) } \\
\hline Surgical resection & $12(36)$ \\
\hline TACE / TARE & $26(79)$ \\
\hline HAIC & $5(15)$ \\
\hline Sorafenib & $31(94)$ \\
\hline Regorafenib & $13(39)$ \\
\hline Lenvatinib & $2(6)$ \\
\hline \multicolumn{2}{|l|}{ Post nivolumab treatment, $n$ (\%) } \\
\hline No treatment & $21(64)$ \\
\hline Resection & $1(3)$ \\
\hline TACE & $2(6)$ \\
\hline Radiation therapy & $3(9)$ \\
\hline Regorafenib & $2(6)$ \\
\hline Cabozantinib & $2(6)$ \\
\hline HAIC & $1(3)$ \\
\hline Systemic chemotherapy & $2(6)$ \\
\hline \multicolumn{2}{|l|}{ Best responses to nivolumab } \\
\hline Complete response & $2(6)$ \\
\hline Partial response & $4(12)$ \\
\hline Stable disease & $4(12)$ \\
\hline Progressive disease & $19(58)$ \\
\hline Not assessed & $4(12)$ \\
\hline
\end{tabular}

AFP, alpha fetoprotein; ALBI grade, albumin-bilirubin grade; BCLC stage, BarcelonaClinic liver cancer stage; HAIC, hepatic arterial infusion chemotherapy; HBsAg, hepatitis B surface antigen; HCV, hepatitis C; RT, radiotherapy; TACE, transarterial chemoembolization; TARE, transarterial radioembolization.

to establish prognostic factors of OS. For the univariate analyses, such as survival probabilities, the Kaplan-Meier method and log-rank tests were used. Factors that were significant in the univariate analysis at $P<0.05$ were advanced to the multivariate analysis, which was undertaken using a Cox regression model.

\section{RESULTS}

\section{Study Cohort Demographics}

As indicated in Table 1, the study involved a total of 33 patients, 25 of whom were male (76\%). Ages ranged from 37-79 years, with a median of 57 years. A majority of patients $(88 \%)$ had been assigned stage $\mathrm{C}$ on the Barcelona Clinic Liver Cancer (BCLC) staging system, with a median tumor size of $3.5 \mathrm{~cm}$. Extrahepatic metastases were reported in 26 patients (79\%), and portal vein tumor thrombosis were detected in 10 patients (30\%). The most prevalent underlying liver diseases was chronic HBV infection, which affected 29 individuals (88\%). A majority of participants (79\%) were classified as Child-Pugh class A at the time of enrolment, and 15 patients (45\%) were ALBI grade 1. The median level of AFP was $665 \mathrm{ng} / \mathrm{mL}$ (normal range: $<8.1 \mathrm{ng} / \mathrm{mL}$ ), and the level of 17 patients $(52 \%)$ were above $1000 \mathrm{ng} / \mathrm{mL}$. Most of the enrolled patients (94\%) underwent sorafenib treatment, and 13 patients (39\%) patients underwent regorafenib treatment prior to the nivolumab therapy. Prior to the systemic therapy, most patients had undergone local-regional therapies such as trans-arterial chemoembolization or hepatic arterial infusion chemotherapy. Eleven patients received further treatments after nivolumab, which included cabozantinib and regorafenib. In this cohort, there was no evidence of a high incidence of immunotherapy-related adverse events. Grade 3 toxicities occurred in one patient, who developed pneumonitis after 5 cycles of nivolumab treatment.

\section{Treatment Responses to Nivolumab}

In this study, the nivolumab monotherapy was administered for 2 to 160 weeks, with a median of 8 weeks, and the number of treatment cycles varied from 1 to 78 , with a median to 3 cycles. In a best response evaluation after nivolumab administration, 2 patients exhibited a CR according to the mRECIST (Table 1). Four patients displayed a PR and 4 patients displayed a SD. However, 4 patients did not undergo imaging for the response evaluation. The objective response rate was $18 \%$ among all the patients that enrolled in this study, and $24 \%$ among patients with evaluable images. The median duration of treatment responses by nivolumab was 13.3 months. The disease control rate was $30 \%$ in our cohort. A waterfall plot describes the marked reductions in target lesions from baseline tumor burden in patients with objective responses to nivolumab monotherapy (Figure 1).

\section{Factors Associated With the Overall Survival}

The median follow-up period after initiation of treatment was 12.5 months, and the OS ranged from 2.3 to 175.1 weeks (median: 26.4 weeks). At the time when data analysis was performed (February 2020), 18 of the 33 patients (55\%) had died from causes such as tumor progression, variceal bleeding, or fatal systemic infection. Figure 2A indicates a significantly better OS for individuals with controlled diseases $(\mathrm{CR}+\mathrm{PR}+\mathrm{SD})$ than for those who displayed PD (log rank test, $P<0.001)$. Figure $2 B$ indicates a significantly better OS for individuals with a maximal tumor size of $<5 \mathrm{~cm}(P=0.002)$, although AFP level did not have a significant impact on the patient survival (Figure 2C). 




FIGURE 1 | Changes in target lesions from the baseline after nivolumab monotherapy. Dashed lines represent a $20 \%$ increase or a $30 \%$ reduction. The percentage changes more than 100\% were truncated to 100\% (asterisks).

Figure 2D indicates a better OS for individuals with ALBI grade 1 than for those with grade 2 with $P=0.004$. Patients with ChildPugh score 5 also showed superior OS to those with score 6 (Figure 2E, $P=0.035$ ).

The prognostic factors for OS after nivolumab treatment are presented in Table 2. These parameters were subjected to univariate analyses initially, and tumor size / ALBI grade were included in a subsequent multivariate Cox regression model. The favorable prognostic factors for OS were a tumor size $<5 \mathrm{~cm}$, the assignment of ALBI grade 1, and ChildPugh score 5 in univariate analyses. In multivariate analysis, tumor size $<5 \mathrm{~cm}(\mathrm{HR}=0.269 ; P=0.034)$ and ALBI grade $1(\mathrm{HR}=0.312 ; P=0.04)$ were both significant factors for OS.

\section{Differential Responses to Nivolumab Among Multiple Tumors in Each Patient}

Due to the previous demonstration that maximal tumor size is a critical factor of OS of patients with nivolumab treatment, we measured response of each tumor among multiple tumors in a single patient. Heterogeneous responses were detected in 6 patients among the 33 enrolled patients (18\%) (Table 3). For patient \#2, the different tumor responses between lung $(1.2 \mathrm{~cm})$ and peritoneal metastasis $(4.3 \mathrm{~cm})$ and the heterogeneous responses to nivolumab treatment was also noted even within the single peritoneal metastatic nodule. This case was previously reported by our group (16). In the peritoneal metastatic nodule, metastatic HCC with partial necrosis was present with viable tumor cells with various types of tumor-infiltrating immune cells, suggesting the immune heterogeneity within a single tumor when it exhibits a considerable size. Figure 3 shows the imaging findings of patient \#6 after 4 cycles of nivolumab. Intrahepatic infiltrative tumor (Figure 3A) showed slight increase in its extent in hepatobiliary phase of primovist-enhanced magnetic resonance imaging, while metastatic nodules in lung showed dramatic responses after nivolumab (Figure 3B).

\section{DISCUSSION}

This is the first report in Korea demonstrating the potential predictors of OS in patients treated with nivolumab for unresectable HCC. In this study, we investigated the safety, efficacy, and the potential predictors of OS in nivolumab monotherapy for unresectable HCC in an endemic area of HBV infection. Moreover, we demonstrated the striking heterogeneous responses to nivolumab monotherapy in a single patient with multiple tumors according to each tumor size. The median OS of the participating patients (26.4 weeks) was longer than the recently conducted real-world study in Europe (34 enrolled patients, OS: 7.5 weeks), which included many patients with poor liver function (41\% with Child Pugh class B) (23), but shorter than the Taiwanese real-world study (92 patients with nivolumab treatment and 3 patients with pembrolizumab treatment, OS: 11.9 months) (24). As expected, it was also shorter than that of the ITT analysis comprising an Asian cohort of CheckMate-040 (14.9 months) (14). As nivolumab is approved only in patients with previous sorafenib failure in Korea and not reimbursed by the government insurance system, Korean HCC patients receive nivolumab treatment as the last possible option for the advanced HCC. Furthermore, shorter follow-up duration may also have affected the shorter OS of our real-world data than those of previous clinical trial data.

In our study, maximal tumor diameter was the significant pre-treatment factor that affected the OS in multivariate analyses. Previous report demonstrated that the ratio of Tcell invigoration to tumor burden ratio correlates with the response to pembrolizumab in melanoma patients $(25,26)$. Other clinical studies showed that tumor size is an independent factor for OS in melanoma and non-small cell lung cancer patients treated with nivolumab or pembrolizumab (27-29). In HCC, very recent data using multi-omics approaches demonstrated the significant heterogeneity of tumor cells in HCC, while the heterogeneity of immune microenvironment was not as dramatic (15). In line with these reports, differential responses to nivolumab among multiple tumors in a patient with HCC can be understood. Heterogeneous responses to nivolumab among multiple metastatic tumors were also demonstrated in melanoma (30) and non-small cell lung cancer patients (31). This heterogeneity can be explained by innate or acquired resistance of tumor cells when a considerable tumor burden exists. In this report, we observed that each tumor size in a patient with multiple tumors may be associated with the heterogeneous responses to nivolumab. In HCC, immune heterogeneity may be applied to the larger tumors that may contain the higher number of resistant clones to immune checkpoint inhibitors. To overcome this heterogeneity, there have been studies investigating the possible synergic benefits for advance HCC of combination therapy (32). Lenvatinib plus pembrolizumab or bevacizumab plus atezolizumab has demonstrated promising objective response rates (ORRs). A study at the European Society for Medical Oncology Asia Congress 2019 showed significant improvements of atezolizumab and bevacizumab over sorafenib 

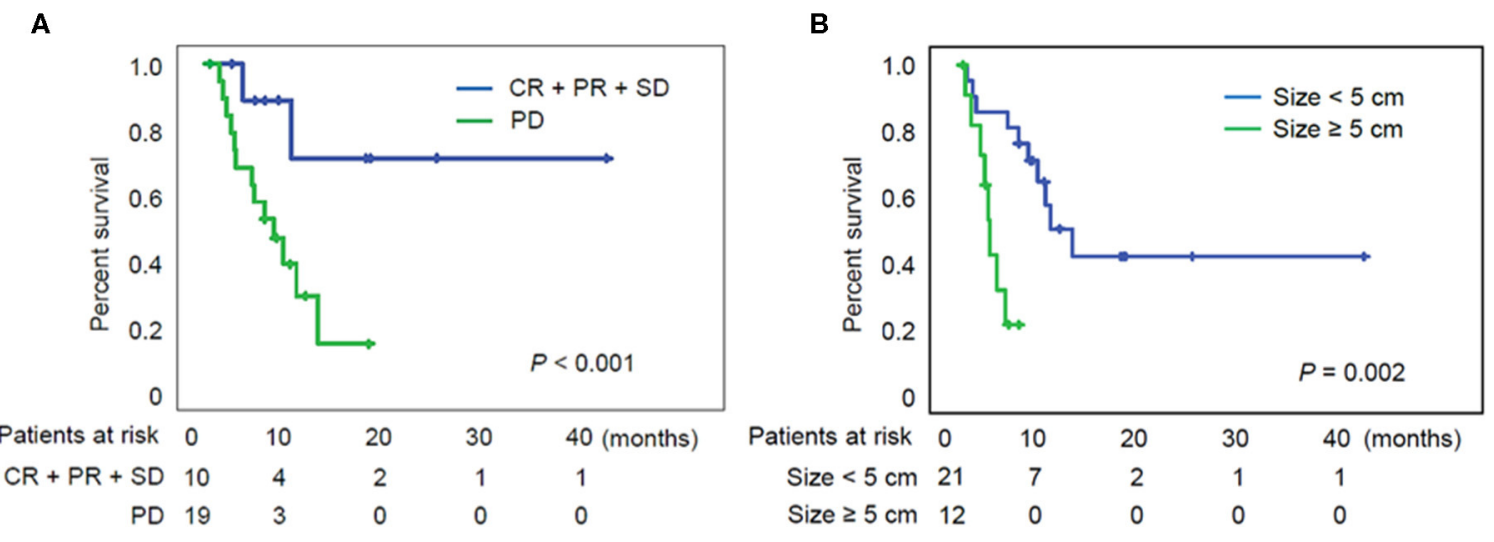

C

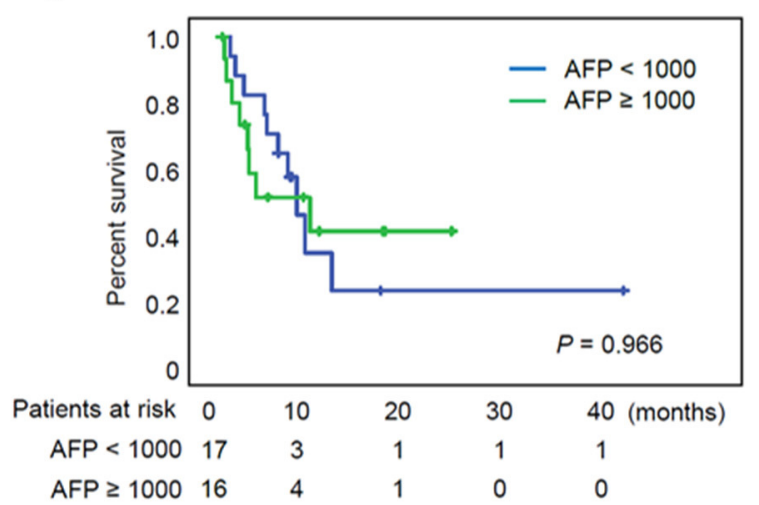

D

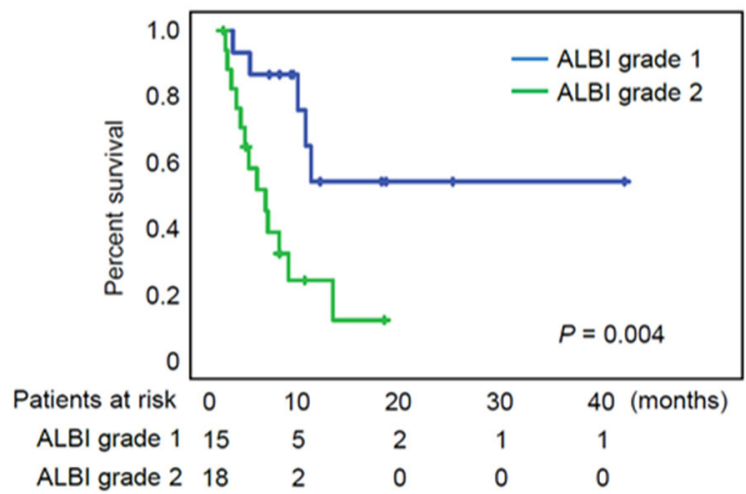

E

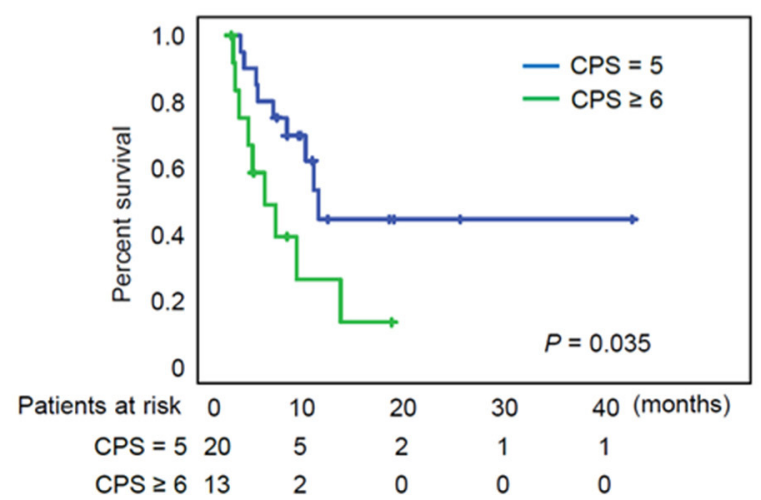

FIGURE 2 | Overall survival of patients according to the various clinical parameters. (A) Overall survival of patients according to the tumor response. (B) Overall survival of patients according to the tumor size. (C) Overall survival of patients according to the AFP. (D) Overall survival of patients according to the ALBI grade. (E) Overall survival of patients according to the CPS. AFP, alpha fetoprotein; ALBI grade, albumin-bilirubin grade; CPS, Child-Pugh score; CR, complete response; PD, progressive disease; PR, partial response; SD, stable disease.

in OS and recurrence-free survival for unresectable HCC (phase 3 IMbrave 150) (32). This suggests that resistance to immune checkpoint inhibitors can be overcome by the combination with anti-angiogenic drugs or tyrosine-kinase inhibitors.

Patients in the real-world cohorts are typically more heterogeneous than those recruited into clinical trials. It is currently still unclear whether nivolumab offers any OS benefit to patients with decreased liver function. There was also a limited treatment effect to these patients in this study. The data from our cohort confirmed the ALBI grade as an independent survival predictor in patients undergoing nivolumab treatment. The results of our survival analysis indicate that ALBI grade 1 is an independent factor for the favorable survival. This is in accord with the previous study demonstrating the 
TABLE 2 | Factors associated with overall survival in 33 patients treated with nivolumab monotherapy.



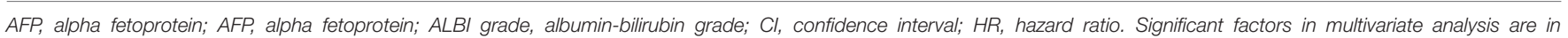
bold characters.

TABLE 3 | Patients with heterogeneous responses to nivolumab.

\begin{tabular}{|c|c|c|c|c|c|c|c|c|c|}
\hline \multirow[b]{2}{*}{ Pt. } & \multicolumn{2}{|c|}{ Intrahepatic tumor } & \multicolumn{3}{|c|}{ Extrahepatic tumor-1 } & \multicolumn{3}{|c|}{ Extrahepatic tumor-2 } & \multirow[t]{2}{*}{ Overall response } \\
\hline & Size $(\mathrm{cm})$ & Response & Location & Size $(\mathrm{cm})$ & Response & Location & Size $(\mathrm{cm})$ & Response & \\
\hline$\# 1$ & \multicolumn{2}{|c|}{ No tumor } & Lung & 4.2 & $10 \% \uparrow$ & Lung & 2.6 & $60 \% \downarrow$ & $P R$ \\
\hline \#2 & \multicolumn{2}{|c|}{ No tumor } & Peritoneum & 4.3 & $13 \% \uparrow$ & Lung & 1.2 & $80 \% \downarrow$ & PR \\
\hline \#3 & 1.4 & $5 \% \downarrow$ & Lung & 1.8 & $10 \% \uparrow$ & Lung & 1.2 & $30 \% \downarrow$ & $\mathrm{SD}$ \\
\hline$\# 4$ & 3.3 & $100 \% \uparrow$ & Lung & 1.2 & $70 \% \downarrow$ & Lung & 1 & $80 \% \downarrow$ & $\mathrm{SD}$ \\
\hline \#5 & 3.3 & $25 \% \downarrow$ & Peritoneum & 1.0 & $200 \% \uparrow$ & Lung & 1 & $100 \% \uparrow$ & PD \\
\hline \#6 & 7.2 & $5 \% \uparrow$ & Lung & 2.1 & $55 \% \downarrow$ & Lung & 1.9 & $62 \% \downarrow$ & PR \\
\hline
\end{tabular}

$P D$, progressive disease; $P R$, partial response; $S D$, stable disease.

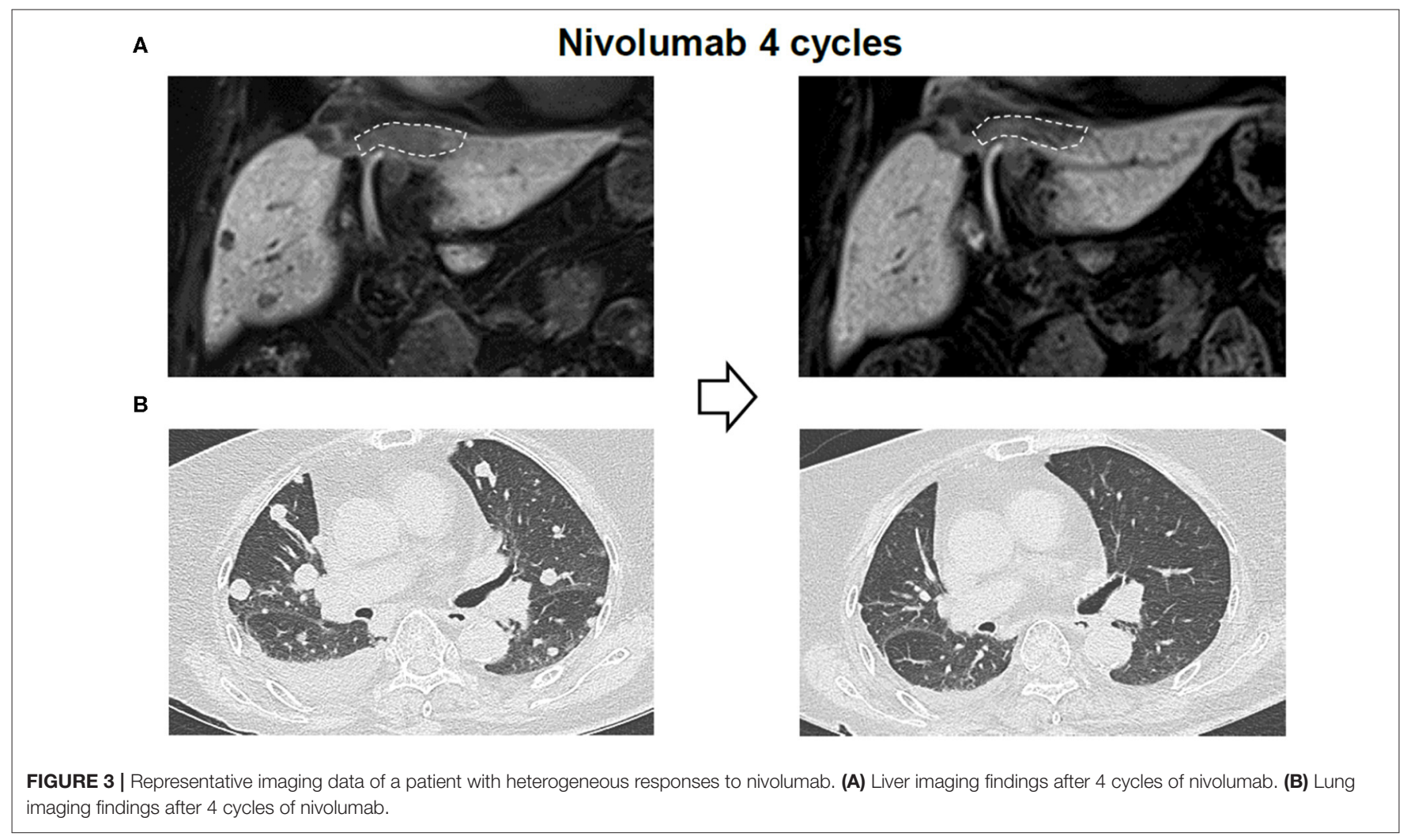


survival-predictable ability of the ALBI grade at the time of sorafenib discontinuation (33).

This study has a number of limitations. This was a small-sized retrospective study that used patients only from three facilities. Such a small number of the cohort is not sufficient to validate the safety and efficacy of the drug. Moreover, regular tumor reassessment by clinical and imaging evaluation would have decreased the observation bias. Lastly, since liver biopsy was not performed routinely before nivolumab treatment, the molecular biomarkers for nivolumab responses were not studied.

In conclusion, our study demonstrates that nivolumab monotherapy is clinically efficacious in treating unresectable $\mathrm{HCC}$ in an endemic area of HBV infection. Maximal tumor diameter and the indicator of liver function (ALBI grade) were significant factors in multivariate analyses that predicted the OS of HCC patients treated with nivolumab monotherapy. Future prospective study is required to overcome the probable heterogeneous efficacy of nivolumab monotherapy according to each tumor size.

\section{DATA AVAILABILITY STATEMENT}

The original contributions presented in the study are included in the article/supplementary materials, further inquiries can be directed to the corresponding author/s.

\section{REFERENCES}

1. Yang JD, Hainaut P, Gores GJ, Amadou A, Plymoth A, Roberts LR. A global view of hepatocellular carcinoma: trends, risk, prevention and management. Nat Rev Gastroenterol Hepatol. (2019) 16:589-604. doi: 10.1038/s41575-019-0186-y

2. Sung PS, Yang K, Bae SH, Oh JS, Chun HJ, Nam HC, et al. Reduction of intrahepatic tumour by hepatic arterial infusion chemotherapy prolongs survival in hepatocellular carcinoma. Anticancer Res. (2019) 39:390916. doi: 10.21873/anticanres. 13542

3. Sung PS, Park HL, Yang K, Hwang S, Song MJ, Jang JW, et al. (18)Ffluorodeoxyglucose uptake of hepatocellular carcinoma as a prognostic predictor in patients with sorafenib treatment. Eur J Nucl Med Mol Imaging. (2018) 45:384-91. doi: 10.1007/s00259-017-3871-5

4. Ogasawara S, Ooka Y, Koroki K, Maruta S, Kanzaki H, Kanayama $\mathrm{K}$, et al. Switching to systemic therapy after locoregional treatment failure: Definition and best timing. Clin Mol Hepatol. (2020) 26:15562. doi: 10.3350/cmh.2019.0021n

5. Hilmi M, Neuzillet C, Calderaro J, Lafdil F, Pawlotsky JM, Rousseau B. Angiogenesis and immune checkpoint inhibitors as therapies for hepatocellular carcinoma: current knowledge and future research directions. J Immunother Cancer. (2019) 7:333. doi: 10.1186/s40425-0190824-5

6. Finn RS, Ryoo BY, Merle P, Kudo M, Bouattour M, Lim HY, et al. Pembrolizumab as second-line therapy in patients with advanced hepatocellular carcinoma in KEYNOTE-240: a randomized, double-blind, phase III trial. J Clin Oncol. (2020) 38:193-202. doi: 10.1200/JCO.19.01307

7. Zhu AX, Finn RS, Edeline J, Cattan S, Ogasawara S, Palmer D, et al. Pembrolizumab in patients with advanced hepatocellular carcinoma previously treated with sorafenib (KEYNOTE-224): a non-randomised, open-label phase 2 trial. Lancet Oncol. (2018) 19:940-952. doi: 10.1200/JCO.2018.36.4_suppl.209

8. El-Khoueiry AB, Sangro B, Yau T, Crocenzi TS, Kudo M, Hsu $\mathrm{C}$, et al. Nivolumab in patients with advanced hepatocellular carcinoma (CheckMate 040): an open-label, non-comparative,

\section{ETHICS STATEMENT}

The studies involving human participants were reviewed and approved by the Institutional Review Board of the Catholic University of Korea (Xc20RIDI0015). The patients/participants provided their written informed consent to participate in this study.

\section{AUTHOR CONTRIBUTIONS}

PS: study design, data collection, data analysis, data interpretation, article, and article approval. JL, SKL, HL, HY, HN, and SWL: data collection. JJ, SB, JC, $\mathrm{NH}$, and SY: data interpretation and article approval. All authors contributed to the article and approved the submitted version.

\section{FUNDING}

This research was supported by the Basic Science Research Program through the National Research Foundation of Korea (NRF) and funded by the Ministry of Education (NRF2019R1I1A1A01059642) (PS).

phase $1 / 2$ dose escalation and expansion trial. Lancet.

(2017) 389:2492-502. doi: 10.1016/S0140-6736(17)31046-2

9. Yao X, Wang L, Gao J. Overshadowed prospect of programmed cell death protein-1 (PD-1) inhibitor as monotherapy for patients with advanced hepatocellular carcinoma. Biosci Trends. (2019) 13:282-3. doi: 10.5582/bst.2019.01161

10. Bangaru S, Marrero JA, Singal AG. Review article: new therapeutic interventions for advanced hepatocellular carcinoma. Aliment Pharmacol Ther. (2020) 51:78-89. doi: 10.1111/apt.15573

11. Xu W, Liu K, Chen M, Sun JY, McCaughan GW, Lu XJ, et al. Immunotherapy for hepatocellular carcinoma: recent advances and future perspectives. Ther Adv Med Oncol. (2019) 11:1758835919862692. doi: 10.1177/1758835919862692

12. Keenan BP, Fong L, Kelley RK. Immunotherapy in hepatocellular carcinoma: the complex interface between inflammation, fibrosis, and the immune response. J Immunother Cancer. (2019) 7:267. doi: 10.1186/s40425-0190749-z

13. Vitale A, Trevisani F, Farinati F, Cillo U. Treatment of hepatocellular carcinoma in the Precision Medicine era: from treatment stage migration to therapeutic hierarchy. Hepatology. (2020). doi: 10.1002/hep.31187. [Epub ahead of print].

14. Yau T, Hsu C, Kim TY, Choo SP, Kang YK, Hou MM, et al. Nivolumab in advanced hepatocellular carcinoma: sorafenib-experienced asian cohort analysis. J Hepatol. (2019) 71:543. doi: 10.1016/j.jhep.2019.05.014

15. Zhang Q, Lou Y, Yang J, Wang J, Feng J, Zhao Y, et al. Integrated multiomic analysis reveals comprehensive tumour heterogeneity and novel immunophenotypic classification in hepatocellular carcinomas. Gut. (2019) 68:2019-31. doi: 10.1136/gutjnl-2019-318912

16. Yang H, Sung PS, Lee J, Yoon SK, Jung ES, Park CS, et al. Heterogenous responses to nivolumab in a single metastatic nodule in hepatocellular carcinoma: role of salvage surgery. Hepatobiliary Surg Nutr. (2019) 8:56971. doi: 10.21037/hbsn.2019.08.10

17. Kim TH, Kim SY, Tang A, Lee JM. Comparison of international guidelines for noninvasive diagnosis of hepatocellular carcinoma: 2018 update. Clin Mol Hepatol. (2019) 25:245-63. doi: 10.3350/cmh.2018.0090 
18. e.e.e. European Association for the Study of the Liver. Electronic address, L. European association for the study of the, easl clinical practice guidelines: management of hepatocellular carcinoma. J Hepatol. (2018) 69:182-236. doi: 10.1016/j.jhep.2018.03.019

19. Johnson PJ, Berhane S, Kagebayashi C, Satomura S, Teng M, Reeves HL, et al. Assessment of liver function in patients with hepatocellular carcinoma: a new evidence-based approach-the ALBI grade. J Clin Oncol. (2015) 33:5508. doi: 10.1200/JCO.2014.57.9151

20. Puzanov I, Diab A, Abdallah K, Bingham CO, 3rd, Brogdon C, Dadu $\mathrm{R}$, et al. Society for immunotherapy of cancer toxicity management working, managing toxicities associated with immune checkpoint inhibitors: consensus recommendations from the society for immunotherapy of cancer (SITC) toxicity management working group. J Immunother Cancer. (2017) 5:95. doi: 10.1186/s40425-017-0300-Z

21. Sangro B, Chan SL, Meyer T, Reig M, El-Khoueiry A, Galle PR. Diagnosis and management of toxicities of immune checkpoint inhibitors in hepatocellular carcinoma. J Hepatol. (2020) 72:320-41. doi: 10.1016/j.jhep.2019.10.021

22. Lencioni R, Llovet JM. Modified RECIST (mRECIST) assessment for hepatocellular carcinoma. Semin Liver Dis. (2010) 30:5260. doi: 10.1055/s-0030-1247132

23. Finkelmeier F, Czauderna C, Perkhofer L, Ettrich TJ, Trojan J, Weinmann A, et al. Feasibility and safety of nivolumab in advanced hepatocellular carcinoma: real-life experience from three German centers. J Cancer Res Clin Oncol. (2019) 145:253-9. doi: 10.1007/s00432-018-2780-8

24. Lee PC, Chao Y, Chen MH, Lan KH, Lee CJ, Lee IC, et al. Predictors of response and survival in immune checkpoint inhibitor-treated unresectable hepatocellular carcinoma. Cancers (Basel). (2020) 12:182. doi: $10.3390 /$ cancers 12010182

25. Huang AC, Postow MA, Orlowski RJ, Mick R, Bengsch B, Manne S, et al. Tcell invigoration to tumour burden ratio associated with anti-PD-1 response. Nature. (2017) 545:60-5. doi: 10.1038/nature22079

26. O'Donnell JS, Teng MWL, Smyth MJ. Cancer immunoediting and resistance to T cell-based immunotherapy. Nat Rev Clin Oncol. (2019) 16:15167. doi: 10.1038/s41571-018-0142-8

27. Nakamura Y. Biomarkers for immune checkpoint inhibitor-mediated tumor response and adverse events. Front Med (Lausanne). (2019) 6:119. doi: 10.3389/fmed.2019.00119
28. Joseph RW, Elassaiss-Schaap J, Kefford R, Hwu WJ, Wolchok JD, Joshua $\mathrm{AM}$, et al. Baseline tumor size is an independent prognostic factor for overall survival in patients with melanoma treated with pembrolizumab. Clin Cancer Res. (2018) 24:4960-7. doi: 10.1158/1078-0432.CCR-18-3340

29. Katsurada M, Nagano T, Tachihara M, Kiriu T, Furukawa K, Koyama K, et al. Baseline tumor size as a predictive and prognostic factor of immune checkpoint inhibitor therapy for non-small cell lung cancer. Anticancer Res. (2019) 39:815-25. doi: 10.21873/anticanres.13180

30. Pires da Silva I, Lo S, Quek C, Gonzalez M, Carlino MS, Long GV, et al. Site-specific response patterns, pseudoprogression, and acquired resistance in patients with melanoma treated with ipilimumab combined with anti-PD-1 therapy. Cancer. (2020) 126:86-97. doi: 10.1002/cncr. 32522

31. Morita R, Okishio K, Shimizu J, Saito H, Sakai H, Kim YH, et al. Real-world effectiveness and safety of nivolumab in patients with non-small cell lung cancer: A multicenter retrospective observational study in Japan. Lung Cancer. (2020) 140:8-18. doi: 10.1016/j.lungcan.2019.11.014

32. Cheng AL, Hsu C, Chan SL, Choo SP, Kudo M. Challenges of combination therapy with immune checkpoint inhibitors for hepatocellular carcinoma. J Hepatol. (2020) 72:307-19. doi: 10.1016/j.jhep.2019. 09.025

33. Pinato DJ, Yen C, Bettinger D, Ramaswami R, Arizumi T, Ward C, et al. The albumin-bilirubin grade improves hepatic reserve estimation post-sorafenib failure: implications for drug development. Aliment Pharmacol Ther. (2017) 45:714-22. doi: 10.1111/apt.13904

Conflict of Interest: The authors declare that the research was conducted in the absence of any commercial or financial relationships that could be construed as a potential conflict of interest.

Copyright (c) 2020 Sung, Jang, Lee, Lee, Lee, Yang, Nam, Lee, Bae, Choi, Han and Yoon. This is an open-access article distributed under the terms of the Creative Commons Attribution License (CC BY). The use, distribution or reproduction in other forums is permitted, provided the original author(s) and the copyright owner(s) are credited and that the original publication in this journal is cited, in accordance with accepted academic practice. No use, distribution or reproduction is permitted which does not comply with these terms. 